\title{
An enhanced vector-free allele exchange (VFAE) mutagenesis protocol for genome editing in a wide range of bacterial species
}

(a) CrossMark

\author{
Ahmed E. Gomaa, Chen Zhang, Zhimin Yang, Liguo Shang, Shijie Jiang, Zhiping Deng, Yuhua Zhan, Wei Lu, \\ Min Lin and Yongliang Yan*
}

\begin{abstract}
Vector-free allele exchange (VFAE) is a newly developed protocol for genome editing in Pseudomonas species. Although several parameters have been determined to optimize the procedures for obtaining a stable and highfrequency mutation, numerous false-positive clones still appear on the plate, which increases the difficulty of finding the desired mutants. It has also not been established whether this protocol can be used for genome editing in other bacterial species. In the current study, the protocol was modified to dramatically decrease the occurrence of falsepositive colonies using Pseudomonas stutzeri A1501 as a model strain. This improvement was reached by increasing the occurrence of circular-DNA cassettes of the correct size. Furthermore, the enhanced protocol was used to construct mutants in both the gram-negative Escherichia coli BL21 and gram-positive Bacillus subtilis 168 strains. The protocol works well in both strains, yielding ideal results with a low percentage of false-positive colonies. In summary, the enhanced VFAE mutagenesis protocol is a potential tool for use in bacterial genome editing.
\end{abstract}

Keywords: Vector-free allele exchange (VFAE), Homologous recombination, Pseudomonas stutzeri, Escherichia coli, Bacillus subtilis, Genome editing

\section{Introduction}

Gene targeting mediated by homologous recombination (HR) is a powerful tool for functional analyses via reverse genetics. Several tools are available for manipulating the bacterial genomes, the most common of which are based on homologous recombination, including the recombineering and CRISPR-Cas9 methodologies (Liu et al. 2003; Jinek et al. 2012; Boyle et al. 2013; Jiang et al. 2013; Gagnon et al. 2014; Ramakrishna et al. 2014; Zuris et al. 2014; Jiang et al. 2015). A common approach to generate gene replacements in bacteria is a two-step homologous recombination (Johnson et al. 2003; Heap et al. 2012; Fu et al. 2012; Faulds-Pain and Wren 2013).

Vector free allele exchange (VFAE) is a new vectorless protocol that was recently proposed (Fig. 1a) (Gomaa et al. 2017). The approach is based on homologous

${ }^{*}$ Correspondence: yanyongliang@caas.cn

Biotechnology Research Institute, Chinese Academy of Agricultural Sciences, Beijing 100081, People's Republic of China recombination in which a recipient bacterial strain is electroporated with a circularized PCR product carrying an antibiotic resistance cassette that is flanked by homologous DNA fragments of the target locus. The initial experiments were done using non-coding RNA $n c R N A 31$, which is a small RNA from Pseudomonas stutzeri A1501 with a length of 119 bp on the genome, and was suggested to have an indirect role in the nitrogen fixation process of A1501. The original VFAE protocol showed rapid inactivation of selected chromosomal gene(s) without requiring any cloning steps. However, a mismatch of the target locus on the genome affected the VFAE protocol through massive colony-screening to find the desired mutant. False-positive clones carry the antibiotic resistance but show the wild-type pattern in the target locus. Thus, the current study has been carried out to improve mutagenesis by non-enzymatically increasing the correctly sized circular DNA product in the ligation mixture (Fig. 1b). An evaluation of the enhanced VFAE protocol was done using two gram-negative bacteria, $P$. 


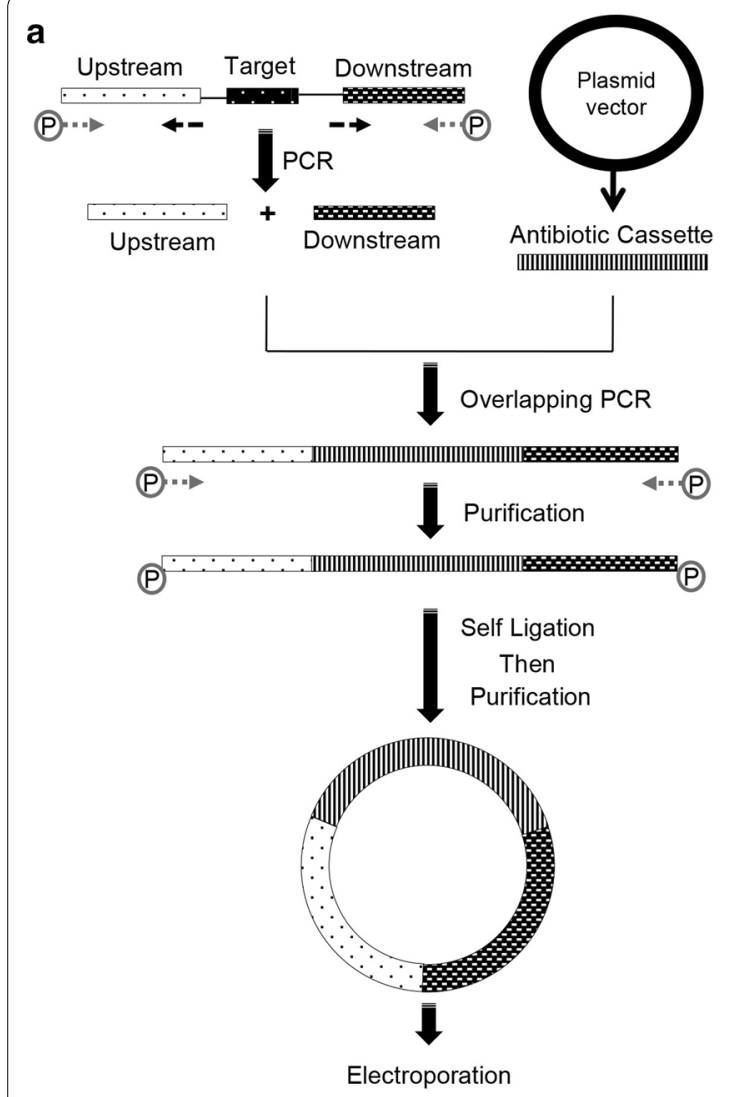

b
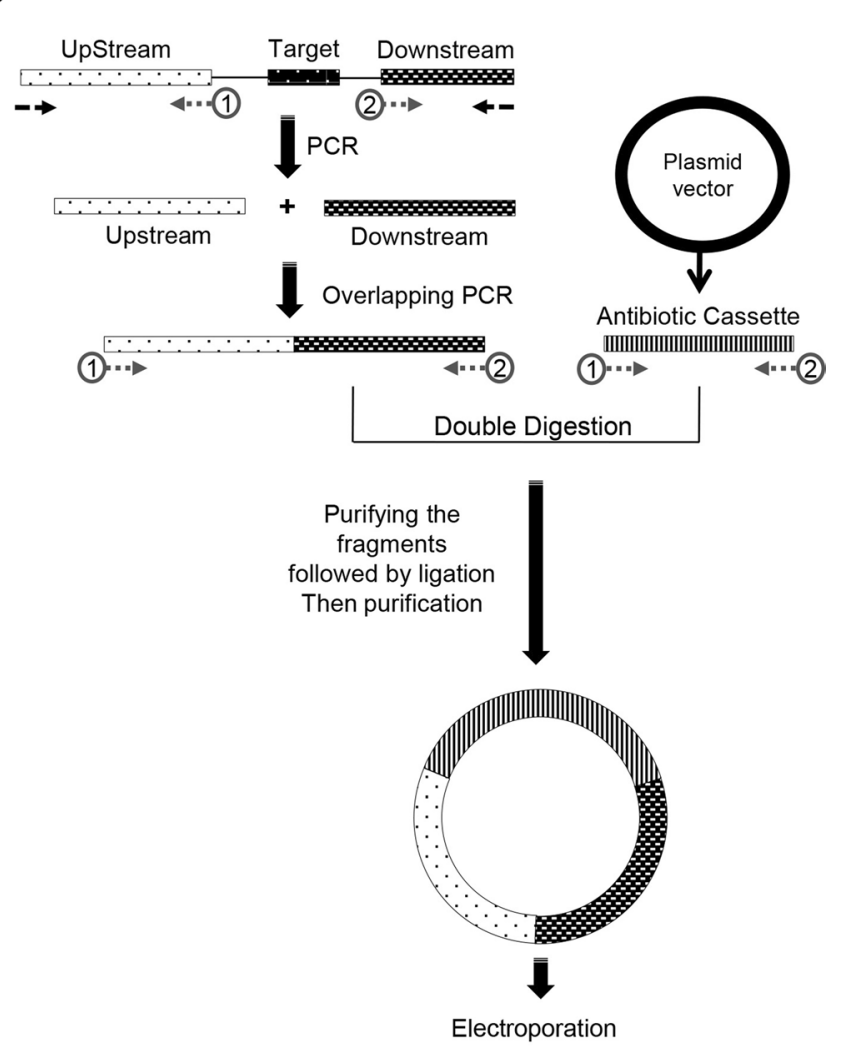

Fig. 1 Schematic representation showing the procedures of the original VFAE protocol (a), and the new modifications of the enhanced protocol (b)

stutzeri A1501 and Escherichia coli BL21, and one grampositive bacterium, Bacillus subtilis 168, to confirm the applicability of the modified approach, which could cover a wide range of bacterial species.

\section{Materials and methods}

\section{Bacterial strains, plasmids, and growth conditions}

Bacterial strains and plasmids used in this study are listed in Additional file 1: Table S1. The P. stutzeri A1501, E. coli BL21 and B. subtilis 168 strains were grown in Luria-Bertani (LB) medium. When necessary, kanamycin $(50 \mu \mathrm{g} / \mathrm{ml})$ was added to the growth media. Bacterial cultures were incubated at $200 \mathrm{rpm}$ and $30{ }^{\circ} \mathrm{C}$ for A1501, and B. subtilis 168 and $E$. coli BL21 were grown at $37^{\circ} \mathrm{C}$.

\section{DNA manipulations}

Genomic and plasmid DNA isolations were performed using the Tiangen Bacterial Genomic Purification Kit (Tiangen, Beijing, China) and the Plasmid Minipreps DNA Purification System (Tiangen, Beijing, China), respectively. Standard PCR amplifications were performed with Prime-Star DNA polymerase (Takara, Beijing, China). The primer locations are illustrated in
Fig. 2a. All of the commercially synthesized oligonucleotides (Tsingke, Beijing, China) used in the study are listed in Additional file 1: Table S2. PCR products were purified using a PCR-Purification Kit (Tiangen, Beijing, China). The obtained overlapping PCR products were sequenced by BGI tech sequencing service (Beijing, China). The double digestion reaction was done using the NEB restriction endonucleases KpnI and SpeI. Ligations of digested and overlapped fragments were performed using $\mathrm{T}_{4}$-ligase (NEB, USA) in four tubes and kept overnight; then, the four tubes were gathered and purified using a PCR purification kit (Tiangen, Beijing, China). The final elution was done using TE buffer and yielded $>60 \mathrm{ng} / \mu \mathrm{l}$. All PCR products were visualized and confirmed by agarose gel electrophoresis. All of the gene cassettes used in the current study are shown in Additional file 1: Table S3.

\section{Electroporation, colony PCR, and sequencing}

Bacterial electrocompetent cells were prepared as follows. The bacterial single colony was taken from fresh LB plate and grown overnight (14-16 h) in LB broth $40 \mathrm{ml}$. Cultures were collected at an $\mathrm{OD}_{600}$ of 1.9-2.0. Cells were pelleted by centrifugation $6000 \mathrm{rpm}$ at $8 \mathrm{~min}$ and then 


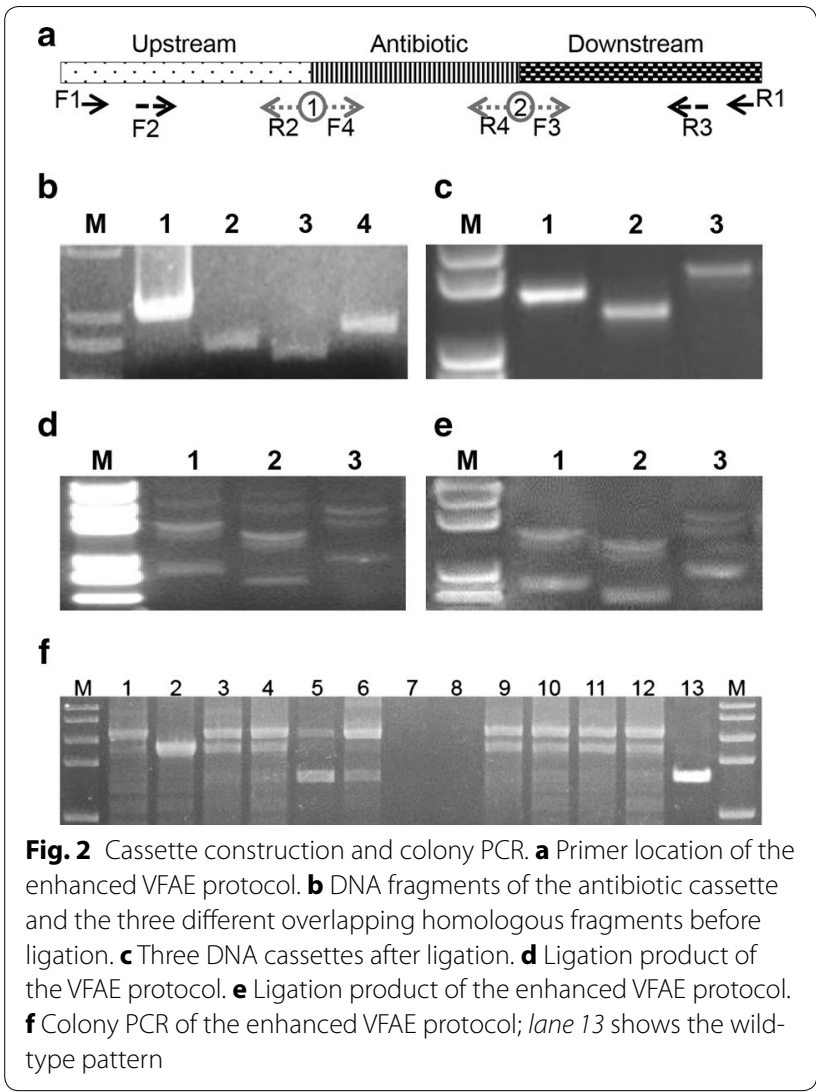

washed 2-3 times with certain buffer for each strain. $300 \mathrm{mM}$ sucrose was used to wash A1501, 10\% glycerol was used to wash BL21. In regards to the washing buffer of $B$. subtilis 168 the composition is $0.5 \mathrm{M}$ sorbitol, $0.5 \mathrm{M}$ mannitol, $10 \%$ glycerol. Finally, cells were resuspended in $1 \mathrm{ml}$ of the same solution used in the washing step. An aliquot of 100-200 $\mu \mathrm{l}$ of the cell suspension was mixed with the recombinant DNA (up to $20 \mu \mathrm{l}$ ). The mixture was placed in a pre-chilled sterile $2 \mathrm{~mm}$ electroporation cuvette and immediately pulsed by use of a Bio-Rad Gene Pulser (Bio-Rad, USA) at the conditions of $2.5 \mathrm{kV}, 200 \mathrm{~W}$, and $25 \mu \mathrm{F}$. The mixture was incubated at $30{ }^{\circ} \mathrm{C}$ (for $P$. stutzeri A1501) or $37^{\circ} \mathrm{C}$ (for E. coli BL21 and B. subtilis 168) overnight with $2 \mathrm{ml}$ of LB broth in $10 \mathrm{ml}$ sterilized test tube with cover. Cells were spread on LB agar containing the appropriate antibiotics and incubated at $30^{\circ} \mathrm{C}$ or $37^{\circ} \mathrm{C}$. Grouped-colony PCR was performed by following the previous protocol (Gomaa et al. 2017). The genedeletion or -disruption was confirmed by PCR and then sequenced using primers S2F, S2R, and S2F as shown in Fig. 2a and Additional file 1: Table S2.

\section{Expression analyses}

A culture of the P. stutzeri A1501 mutant was grown overnight in LB broth supplemented with kanamycin at
$30{ }^{\circ} \mathrm{C}$. RNA isolation was performed with the RNAeasy Bacterial Mini Kit (Qiagen, Germany), followed by RTPCR performed with the First Strand cDNA Transcription Synthesis Kit (PrimeScript RT, Takara, China). The primers used in the first strand synthesis and the PCR amplification are listed in Additional file 1: Table S2. The PCR product was visualized in a 1.0\% agarose gel.

\section{Mutant stability assays}

Five colonies that had been confirmed as deletion mutants of $n c R N A 31$ were purified via streaking three times on selective plates. The colonies were then cultured in $5 \mathrm{ml}$ of LB broth without kanamycin and then incubated at $30{ }^{\circ} \mathrm{C}$. During the following 10 days, $100 \mu \mathrm{l}$ of each culture was diluted in $5 \mathrm{ml}$ of fresh medium daily and incubated for $24 \mathrm{~h}$. On days 1, 5 and 10, all cultures were diluted 100-fold and plated on selective and nonselective plates to determine the frequency of cell viability in terms of the percentage of kanamycin sensitive colonies.

\section{Statistical method}

T-tests were applied to determine the significance between the numbers of colonies that appeared on the selective and non-selective plates of the stability test. All analyses were performed using GraphPad Prism version 5.00 for Windows, GraphPad Software, San Diego California USA.

\section{Result}

Improvement of the VFAE protocol to decrease the occurrence of false-positive colonies

Several parameters were assayed to optimize the procedure of the previous VFAE protocol (Gomaa et al. 2017). However, this protocol has several limitations due to the high frequency of false-positive clones, which increases the difficulty of finding the desired mutants. Therefore, in this study, a modified and enhanced protocol to reduce the percentage of false-positive isolates was developed (Additional file 2). The complete design and comparison of the two approaches is shown in Fig. 1, where Fig. 1a shows the original VFAE protocol (Gomaa et al. 2017), and the new strategy is shown in Fig. 1b. Compared to the phosphorylation of the DNA ends, the main point of this improvement is the addition of two restriction sites (KpnI and SpeI) to the flanks of $n p t$ II and the overlapped homologous fragment (down- and up-stream fragments), which leads to a DNA product with an undetectable concentration of a complete linear DNA cassette after the digestion/ligation reactions. Theoretically, it is based on an increase in the circular DNA with the correct cassette size. The optimum experimental conditions were 
subsequently adapted to the mutagenesis of rpoN gene from E. coli BL21 and the upp gene from B. subtilis 168 .

The optimum conditions used in current study were a DNA homology $>200$ bp and a DNA concentration $>60 \mathrm{ng} / \mu \mathrm{l}$, adjusted according to the previous VFAE protocol (Gomaa et al. 2017). Amplified fragments (ranging from 200 to $600 \mathrm{bp}$ ) of the ncRNA31 locus of the A1501 genome are shown in Additional file 1: Table S2. The homologous ends of the up- and downstream fragments overlapped each other and were flanked by two different restriction sites (KpnI and SpeI). On the opposing ends, the $n p t$ II sequence was also flanked by the same restriction sites (Fig. 2a). After the digestion/ligation reaction, there were three DNA constructs, as shown in Additional file 1: Table S3. The use of four ligation tubes and gathering the ligated product during the purification step increased the number of colonies carrying the DNA cassettes. Figure 1 shows the minor modifications to the VFAE protocol using the restriction sites to connect the marker gene to the homologues fragments, unlike the connection by the overlapping PCR. The minor changes in the cassette construction had a great impact on the number of the false-positive colonies, as shown in Fig. 3; the dramatic decrease in the wild-type pattern (false positive clones) was 0.1 -fold lower than the original VFAE protocol. As shown in Figs. 2f and 3b, almost 90\% mutagenesis was achieved by considering the number of colonies detected by PCR to have the ncRNA31 deletion mutant.

Additionally, an important observation was that the agarose gel of the DNA product from the DNA-circularization step (just after ligation) shows multiple DNA forms represented by several bands with different sizes, as shown in Fig. 2d and e. Moreover, it was found that the new approach of cassette circularization shows fewer band patterns compared to the original VFAE protocol. This suggests that several forms of DNA with different sizes are formed after the ligation step and that the modified protocol led to the reduction of the ligation product patterns.

\section{Expression and mutant stability assays}

Transcription analysis was performed to detect the expression of the $n c R N A 31$ gene in the deletion mutant and the wild-type strain A1501. It was shown that no specific bands appeared in the mutant; however, a specific band was exhibited using the wild-type strain, indicating that the $n c R N A 31$ gene was completely knocked out in the mutant.

To investigate the stability of the mutants, $t$-tests were applied to determine the significance between the numbers of colonies that appeared on the selective and nonselective plates. The analyses showed that the difference was non-significant between the numbers of colonies that appeared on the selective and non-selective plates, as demonstrated with a $P$ value of 0.75 , thus confirming that the deletion mutant was stable.

\section{Comparison of the false positive percentage for the two approaches}

The small non-coding RNA ncRNA31 was used as a model genomic-location to compare the result of the two approaches. The main difference between the approaches is in the circular DNA cassette construction. The original VFAE protocol uses overlapping PCR to connect the three fragments (upstream homologous fragments, downstream homologous fragments, and the antibiotic cassette). In the new modification, a digestion/ligation reaction is used to join the antibiotic resistance cassette to the circular DNA. The original VFAE protocol results
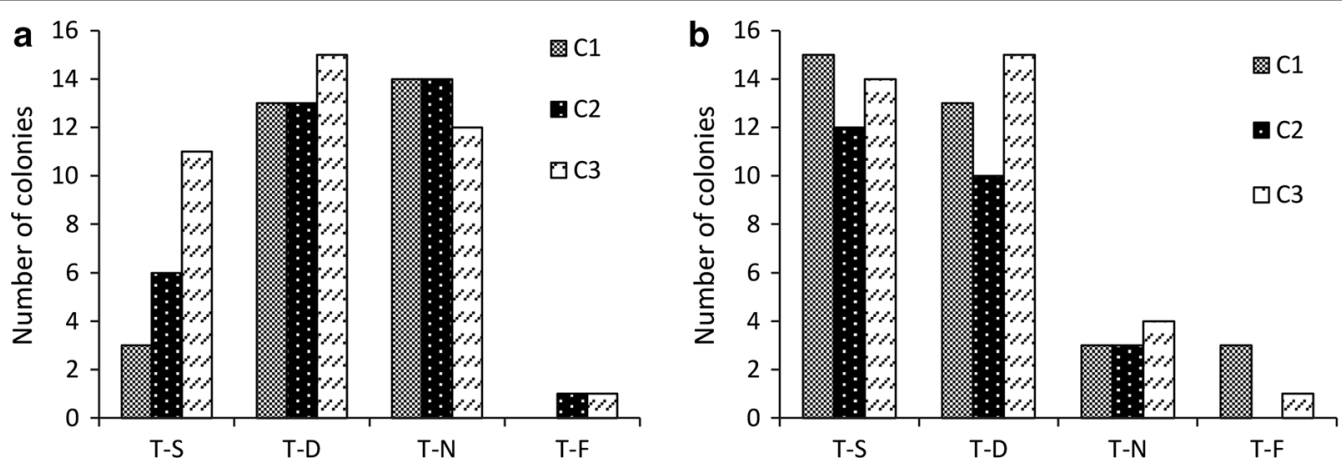

Fig. 3 Graphs showing the number and patterns of bacterial colonies generated on the kanamycin-supplemented plate during the deletion of nCRNA31 from P. stutzeri A1501 using the VFAE protocol. a Number of colonies appearing when the VFAE original protocol was applied to the target locus nCRNA31 (represented in three DNA cassettes with different lengths C1-C3). b Number of colonies appearing when the enhanced VFAE protocol was applied to the target locus nCRNA31 (represented in three DNA cassettes C1-C3). T-S, single crossover mutant; T-D, double crossover mutant; T-N, wild-type pattern. T-F, failed PCR 
in high number of false-positive colonies with average percentage $38 \%$ and has single and double crossover rates of 19 and 39\%, respectively (Fig. 3a). In comparison, the current new modification showed significant improvement in the single and double cross-over, with an average increase of 3.0-fold, especially for the single cross-over (Fig. 3b). For the number of the false-positive clones, the data showed a dramatic decrease of an average of 0.11 -fold. Thus, it appears that the modification of the VFAE protocol impacted the number of false positives, expressed as the average percentages of single and double cross-overs and the number of false positives, at 44,40 , and $10 \%$, respectively.

\section{Extension of the application range of VFAE methods to $E$. coli and B. subtilis}

In order to convince this protocol can be used in other bacterial strains, two widely investigated representative gram-negative strains (E. coli BL21) and a gram-positive strain (B. subtilis 168) were selected as the recipients to construct mutants. The two bacterial species are highly competent strains that have been widely used with suicide vectors for mutagenesis purposes (Dong and Zhang 2014; Gao et al. 2014; Liu et al. 2015; Rahmer et al. 2015; Wenzel and Altenbuchner 2015).

Following the detailed procedures for cassette construction, as mentioned in the material and method section, the two cassettes for two gene locations from bacterial strains (the rpoN gene from E. coli BL21 and the gene upp from $B$. subtilis 168 were also included using $n p t$ II as a marker gene. Only one DNA cassette for each strain was constructed by following the enhanced protocol.

The optimum conditions of the modified protocol (using restriction sites) were replicated at two locations for the two bacterial species, rpoN from E. coli BL21 and upp from B. subtilis 168 . The false positive clones appeared as a single colony from a total of 15 and 13 colonies selected from E. coli BL21 and B. subtilis 168 plates, respectively (shown in Fig. 4a, b). The percentage of the single cross-over events was 53 and $69 \%$ for E. coli BL21 and $B$. subtilis 168 strains, respectively, and the double cross-over percentage was 26 and 15\%, for E. coli BL21 and $B$. subtilis 168 , respectively. Rescreening the colonies for a single crossover on a new plate supplemented with kanamycin showed the double cross over pattern for most of the resulting colonies. The use of this procedure with gram-negative bacteria was our main goal; however, many gram-positive bacteria are also studied and require genome editing tools. The commonly used protocol for genome editing in the model strain B. subtilis 168 is the use of the integrative vector (suicide vector) technique, which has a protocol complexity similar to gram-negative genera (Haijema et al. 1996; Rahmer et al. 2015; Wenzel and Altenbuchner 2015).

\section{Discussion}

To date, the techniques discovered for bacterial genome editing are complex and time-consuming. In E. coli, homologous recombination, site-specific recombination, and transposon-mediated gene transposition techniques are used for chromosomal integration (Martinez-Morales et al. 1999; Court et al. 2002; Song et al. 2010; Song and Lee 2013; Gu et al. 2015). Among these, the VFAE method is considered the simplest and most straightforward protocol for bacterial genome editing and is based on the homologous recombination (Gomaa et al. 2017). However, one of the most common problems that face homologous recombination protocols is the occurrence of false positive clones that frequently appear during the genome editing of bacterial strains, thus increasing the time needed to screen for the desired mutation. Few
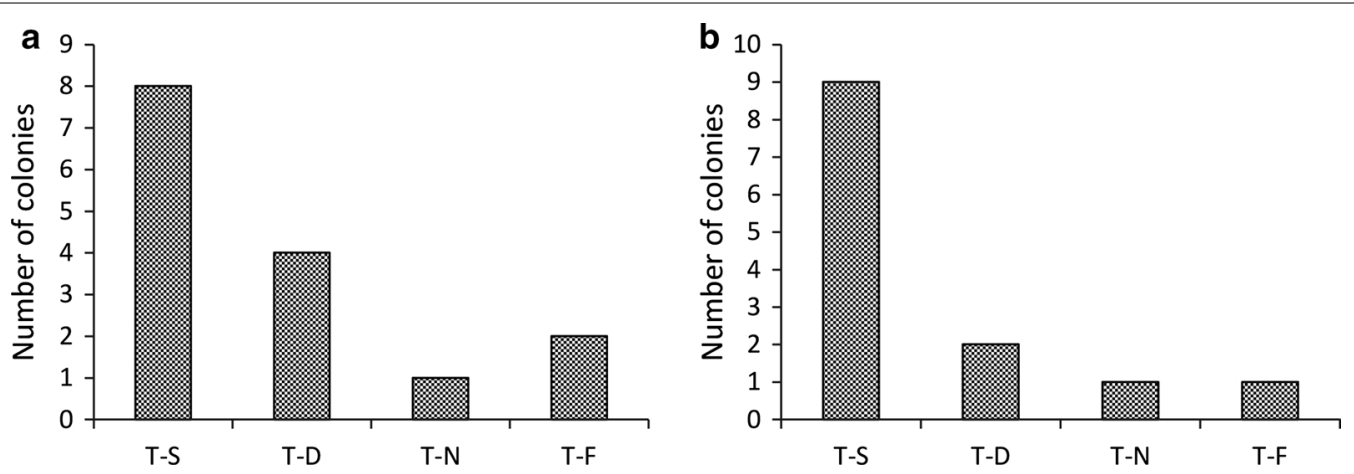

Fig. 4 Graphs showing the number and patterns of the bacterial colonies appearing on the kanamycin-supplemented plates during the deletion of rpoN from E. coli BL21 and upp from B. subtilis 168 using the improved VFAE protocol. a Colonies of E. coli BL21. b Colonies of B. subtilis 168. T-S, single crossover mutant; $T-D$, double crossover mutant; $T-N$, wild-type pattern; $T-F$, failed PCR 
reports addressed the problem of false positive clones, but almost no significant progress has been confirmed (Sabri et al. 2013; Gu et al. 2015). In the current study, the VFAE protocol was enhanced. The new approach was tested and showed a significant improvement in terms of cross-over events and the number of false positive clones. Most of the mutations were in the correct locus and did not have a wild-type pattern. Accordingly, the occurrence of numerous false-positive clones in the original VFAE protocol could be a result of multiple ligations occurring between the same DNA cassettes, leading to the occurrence of multiple forms and sizes of DNA in the same ligation reaction (Additional file 1: Figure S1). Thus, the more we decrease the occurrence of other forms of DNA cassettes with a complete functional resistance gene, the fewer false-positive clones would appear. The ability of cells to bind to and uptake the exogenous DNA is called natural genetic competence. This process has been found in many bacterial species, and it occurs by transporting the environmental DNA fragments through the cell envelope into the cell cytoplasm (Mell and Redfield 2014). Competent cells express proteins that assemble into a complex for DNA-uptake (Chen and Dubnau 2004). The foreign DNA fragments can recombine and replace homologous segments on the chromosome within the competent cell; thus, competence provides cells with a potent mechanism of horizontal gene transfer and access to the nutrients in extracellular DNA (Solomon and Grossman 1996). In the current study, the modified protocol was applied to three different bacterial species (two gram-negative and one gram-positive). In $E$. coli, there are homologues of the competence genes that other species use for DNA uptake and processing. These competence genes in $E$. coli were found to encode for functional uptake machinery, although the amount of transformation cells undergo is limited both by low levels of DNA uptake and by inefficient DNA processing/ recombination (Sinha and Redfield 2012). The development of competence in B. subtilis 168 is part of a complex signal transduction network that is influenced by the level of nutrients in the environment and by the cell density (Ashikaga et al. 2000). The transcriptional factor ComK in B. subtilis 168 can induce the transcription of both recA and comK itself, both of which are essential for the uptake of exogenous DNA in macromolecular form (Grossman 1995; Haijema et al. 1996; Ashikaga et al. 2000). The success that has been achieved with the two bacterial species E. coli BL21 and B. subtilis 168 showed that the VFAE protocol can be renamed as the "vectorless integrative-vector technique". Thus, as long as the integrative vector (suicide vector) technique is functional with any bacterial strain, the VFAE protocol could be widely and successfully applied (Katzen et al. 1999;
Schweizer 2008; Song et al. 2010; Xie et al. 2011; Heap et al. 2012; Sabri et al. 2013; Wang et al. 2015).

\section{Additional files}

Additional file 1: Table S1. Stains and plasmids used in the current study. Table S2. Primers used in the current study. Table S3. DNA-Cassettes construction of the current study. Figure S1. Schematic representation shows the ligation product from the original VFAE procedures, there would be three levels of cassette self-ligations.

Additional file 2. Schematic representation and comparison of the original and modified VFAE procedures.

\section{Abbreviations}

VFAE: vector-free allele exchange; A1501: Pseudomonas stutzeri A1501; Amp: ampicillin; Km: kanamycine; MW: molecular weight; ncRNA: non-coding RNA; OD: optical density; bp: base pair; SD: standard deviation.

\section{Authors' contributions}

YY and ML designed the work; AEG, CZ, ZY and LS performed the research study; SJ and ZD analysis the data; AEG and YZ drafted the manuscript; YY and WL were involved in critically revision the manuscript. All authors read and approved the final manuscript.

\section{Acknowledgements}

We would like to thank Mohammed Shalapy and Zheney Makay for helpful discussions and carefully reading our manuscript.

\section{Competing interests}

The authors declare that they have no competing interests.

\section{Availability of data and materials}

The datasets supporting the conclusions of this article are included within the article and its Additional files 1, 2.

\section{Funding}

This work was supported by Grants from the National Science Foundation of China (31230004, 31470205 and 31470174), National Basic Research Program of China (2015CB755701), Project from the National Research and Development Project of Transgenic Crops of China (2016ZX08009-003), and the Guangdong Innovative and Entrepreneurial Research Team Program (2013S033).

\section{Publisher's Note}

Springer Nature remains neutral with regard to jurisdictional claims in published maps and institutional affiliations.

Received: 7 April 2017 Accepted: 8 June 2017

Published online: 17 June 2017

\footnotetext{
References

Ashikaga S, Nanamiya H, Ohashi Y, Kawamura F (2000) Natural genetic competence in Bacillus subtilis natto OK2. J Bacteriol 182(9):2411-2415

Boyle NR, Reynolds TS, Evans R, Lynch M, Gill RT (2013) Recombineering to homogeneity: extension of multiplex recombineering to large-scale genome editing. Biotechnol J 8:515-522

Chen I, Dubnau D (2004) DNA uptake during bacterial transformation. Nat Rev Microbiol 2:241-249

Court DL, Sawitzke JA, Thomason LC (2002) Genetic engineering using homologous recombination. Annu Rev Genet 36:361-388

Dong H, Zhang D (2014) Current development in genetic engineering strategies of Bacillus species. Microb Cell Fact 13:63
} 
Faulds-Pain A, Wren BW (2013) Improved bacterial mutagenesis by highfrequency allele exchange, demonstrated in Clostridium difficile and Streptococcus suis. Appl Environ Microbiol 79:4768-4771

Fu J, Bian X, Hu S, Wang H, Huang F, Seibert PM, Plaza A, Xia L, Muller R, Stewart AF, Zhang Y (2012) Full-length RecE enhances linear-linear homologous recombination and facilitates direct cloning for bioprospecting. Nat Biotechnol 30:440-446

Gagnon JA, Valen E, Thyme SB, Huang P, Akhmetova L, Ahkmetova L, Pauli A, Montague TG, Zimmerman S, Richter C, Schier AF (2014) Efficient mutagenesis by Cas9 protein-mediated oligonucleotide insertion and large-scale assessment of single-guide RNAs. PLOS ONE 9:e98186

Gao Y, Liu C, Ding Y, Sun C, Zhang R, Xian M, Zhao G (2014) Development of genetically stable Escherichia coli strains for poly(3-hydroxypropionate) production. PLoS ONE 9(5):e97845

Gomaa AE, Deng Z, Yang Z, Shang L, Zhan Y, Lu W, Lin M, Yan Y (2017) Highfrequency targeted mutagenesis in Pseudomonas stutzeri using a vectorfree allele-exchange protocol. J Microbiol Biotechnol 27:335-341

Grossman AD (1995) Genetic Networks controlling the initiation of sporulation and the development of genetic competence in Bacillus subtilis. Rev Genet 29:477-508

Gu P, Yang F, Su T, Wang Q, Liang Q, Qi Q (2015) A rapid and reliable strategy for chromosomal integration of gene(s) with multiple copies. Sci Rep 5:9684

Haijema BJ, Van Sinderen D, Winterling K, Kooistra J, Venema G, Hamoen LW (1996) Regulated expression of the dinR and recA genes during competence development and SOS induction in Bacillus subtilis. Mol Microbiol 22:75-85

Heap JT, Ehsaan M, Cooksley CM, Ng YK, Cartman ST, Winzer K, Minton NP (2012) Integration of DNA into bacterial chromosomes from plasmids without a counter-selection marker. Nucleic Acids Res 40(8):e59

Jiang W, Bikard D, Cox D, Zhang F, Marraffini LA (2013) RNA-guided editing of bacterial genomes using CRISPR-Cas systems. Nat Biotechnol 31:233-239

Jiang Y, Chen B, Duan C, Sun B, Yang J, Yang S (2015) Multigene editing in the Escherichia coli genome using the CRISPR-Cas9 system. Appl Environ Microbiol 81:2506-2514

Jinek M, Chylinski K, Fonfara I, Hauer M, Doudna JA, Charpentier E (2012) A programmable dual-RNA-guided DNA endonuclease in adaptive bacterial immunity. Science 337:816-821

Johnson JR, Lockman HA, Owens K, Jelacic S, Tarr PI (2003) High-frequency secondary mutations after suicide-driven allelic exchange mutagenesis in extraintestinal pathogenic Escherichia coli. J Bacteriol 185:5301-5305

Katzen F, Becker A, lelmini MV, Oddo CG, lelpi L (1999) New mobilizable vectors suitable for gene replacement in gram-negative bacteria and their use in mapping of the $3^{\prime}$ end of the Xanthomonas campestris pv. campestris gum operon. Appl Environ Microbiol 65:278-282

Liu P, Jenkins NA, Copeland NG (2003) A highly efficient recombineeringbased method for generating conditional knockout mutations. Genome Res 13:476-484
Liu Q, Li Y, Zhao X, Yang X, Liu Q, Kong Q (2015) Construction of Escherichia coli mutant with decreased endotoxic activity by modifying lipid A structure. Mar Drugs 13(6):3388-3406

Martinez-Morales F, Borges AC, Martinez A, Shanmugam KT, Ingram LO (1999) Chromosomal integration of heterologous DNA in Escherichia coli with precise removal of markers and replicons used during construction. J Bacteriol 181:7143-7148

Mell JC, Redfield RJ (2014) Natural competence and the evolution of DNA uptake specificity. J Bacteriol 196:1471-1483

Rahmer R, Heravi KM, Altenbuchner J (2015) Construction of a super-competent Bacillus subtilis 168 using the PmtIA-comKS inducible cassette. Front Microbiol. 6:1431

Ramakrishna S, Kwaku Dad A-B, Beloor J, Gopalappa R, Lee S-K, Kim H (2014) Gene disruption by cell-penetrating peptide-mediated delivery of Cas 9 protein and guide RNA. Genome Res 24:1020-1027

Sabri S, Steen JA, Bongers M, Nielsen LK, Vickers CE (2013) Knock-in/Knock-out (KIKO) vectors for rapid integration of large DNA sequences, including whole metabolic pathways, onto the Escherichia coli chromosome at well-characterised loci. Microb Cell Fact 12:60

Schweizer HP (2008) Bacterial genetics: past achievements, present state of the field, and future challenges. Biotechniques 44:633-641

Sinha S, Redfield RJ (2012) Natural DNA uptake by Escherichia coli. PLoS ONE 7(4):e35620

Solomon JM, Grossman AD (1996) Who's competent and when: regulation of natural genetic competence in bacteria. Trends Genet 12:150-155

Song CW, Lee SY (2013) Rapid one-step inactivation of single or multiple genes in Escherichia coli. Biotechnol J 8:776-784

Song J, Dong H, Ma C, Zhao B, Shang G (2010) Construction and functional characterization of an integrative form $\lambda$ red recombineering Escherichia coli strain. FEMS Microbiol Lett. 309(2):178-183

Wang P, Yu Z, Li B, Cai X, Zeng Z, Chen X, Wang X (2015) Development of an efficient conjugation-based genetic manipulation system for Pseudoalteromonas. Microb Cell Fact 14:11

Wenzel M, Altenbuchner J (2015) Development of a markerless gene deletion system for Bacillus subtilis based on the mannose phosphoenolpyruvatedependent phosphotransferase system. Microbiology 161:1942-1949

Xie Z, Okinaga T, Qi F, Zhang Z, Merritt J (2011) Cloning-independent and counterselectable markerless mutagenesis system in Streptococcus mutans. Appl Environ Microbiol 77:8025-8033

Zuris JA, Thompson DB, Shu Y, Guilinger JP, Bessen JL, Hu JH, Maeder ML Joung JK, Chen Z-Y, Liu DR (2014) Cationic lipid-mediated delivery of proteins enables efficient protein-based genome editing in vitro and in vivo. Nat Biotechnol 33:73-80

\section{Submit your manuscript to a SpringerOpen ${ }^{\circ}$ journal and benefit from:}

- Convenient online submission

- Rigorous peer review

- Open access: articles freely available online

- High visibility within the field

- Retaining the copyright to your article

Submit your next manuscript at springeropen.com 